\title{
Comparative Evaluation of Lateral Condylar Guidance Angle Measured Using Hanau's Formula \& Lateral Interocclusal Records
}

\author{
Rajesh Shetty ${ }^{1}$, Sanath Shetty ${ }^{2}$, Naresh Shetty ${ }^{3}$, \\ Siddharth Shrirang Pinge ${ }^{4}$, Mohammed Zahid ${ }^{5}$, Karkala Sayed Suhaim ${ }^{6}$ \\ 1, 2, 3, 4, 5, 6 Department of Prosthodontics, Yenepoya Dental College, Mangalore, Karnataka, India.
}

\section{ABSTRACT}

\section{BACKGROUND}

The goal of registering condylar guidance is to program the articulator for simulating the patient's condylar movements as accurately as possible to save chairside time involved in adjusting the occlusal interferences at the time of fit in. The use of Hanau's formula ( $\mathrm{L}=\mathrm{H} / 8+12$ ) was advocated by Sir Rudolph Hanau in 1930 which he used to calculate lateral condylar guidance (LCG) for adjusting his articulators. But due to the ease of application, the formula is widely used by dentists till date. Jack Stern, Hanau's longterm partner, confided in 1960 that the 'Formula' was never considered to be precise, after 10 years of study (1920 - 1930). Rather it was a point of approximation. There is a lack of uniformity in the literature regarding average condylar inclinations and its comparison to the Hanau formula, therefore a study was undertaken to evaluate and compare the 2 methods used to determine the lateral condylar guidance.

\section{METHODS}

24 completely dentulous participants were included in the study. Two methods were used to obtain the lateral condylar guidance, the first method was obtaining the calculated lateral condylar guidance using the hanau's formula and the second was obtaining the measured lateral condylar guidance using the interocclusal records. Impressions of each participant were made. Protrusive and lateral records were made using interocclusal recording medium (MAARC ${ }^{\circledR}$ Perfect Bite Registration). After completion of facebow transfer, programming of the articulator was done using protrusive record and the horizontal condylar guidance was recorded. The calculated lateral condylar guidance was recorded using the Hanau's formula \& the measured condylar guidance was recorded using right and left lateral records. Both the values obtained were evaluated and compared.

\section{RESULTS}

Mean LCG values obtained using Hanau's formula was 16 degrees for the left side (SD - 1.404) and for the right side it was 16.17 degrees. (SD - 1.694). Mean LCG values obtained using lateral interocclusal records were 20 degrees for the left side (SD 6.691) and 20.08 degrees (SD - 4.926) for the right side.There was a statistically significant difference between lateral condylar guidance records obtained using Hanau's formula and lateral interocclusal records.

\section{CONCLUSIONS}

Application of Hanau formula is helpful to estimate Bennett angle. No doubt the formula is easy to apply and less cumbersome, but when we are working on high precision demanding cases we should try and find better options to record LCG. Making a lateral interocclusal record and then programming the articulator is a more reliable method.

\section{KEY WORDS}

Hanau's Formula, Bennet Angle, Condylar Guidance, Articulators, Interocclusal Records, Lateral Condylar Guidance
Corresponding Author: Dr. Siddharth Pinge, No. 202, Ajit Bldg, Khandelwal Complex, Evershine Nagar, Malad West, Mumbai - 400064, India. E-mail: siddharthpinge@gmail.com

\section{DOI: $10.14260 / j e m d s / 2021 / 395$}

How to Cite This Article:

Shetty $R$, Shetty $S$, Shetty $N$, et al. Comparative evaluation of lateral condylar guidance angle measured using Hanau's Formula \& lateral interocclusal records. J Evolution Med Dent Sci 2021;10(26):19171920, DOI: 10.14260/jemds/2021/395

Submission 24-02-2021,

Peer Review 02-05-2021

Acceptance 07-05-2021,

Published 28-06-2021.

Copyright (c) 2021 Rajesh Shetty et al. This is an open access article distributed under Creative Commons Attribution License [Attribution 4.0 International (CC BY 4.0)] 


\section{BACKGROUND}

A prosthetic rehabilitation's ultimate objective is to create a prosthesis that is harmonious with the stomatognathic system of the patient. The most important factor in a patient's oral rehabilitation is to assess the path of the condylar inclination. Condylar path is defined as the path traversed by condyle in relation to the articular eminence when the patient's mandible is moved either in protrusive or in the lateral direction from centric relation. Condylar guidance is the mechanical feature of an articulator that regulates the motion of its mobile member in the upper posterior region. ${ }^{1}$ The aim of making interocclusal records is to set the articulator condylar elements such that they replicate inclinations similar to that of the articulation of the temporomandibular joint of the patient, thus eliminating intervention during mandibular articulation.

It is well understood that horizontal and lateral condylar inclinations have great influence on tooth morphology. Horizontal condylar inclination was found to be in proportion with cusp height. Increased horizontal condylar inclination would result in an increased cusp height of the teeth and vice versa, whereas the effects of immediate side shift and progressive side shift would affect the grooves direction and grooves - cusps relation. ${ }^{2}$ Semi adjustable articulator programming is an additional step that is considered mandatory for the construction of removable or fixed prothesis which is precise. Adjusting the horizontal condylar guidance, lateral condylar guidance, and incisal guidance on the semi - adjustable articulator is referred to as programming. This angle differs from one person to the other, as well as from one side to the other. The purpose of registering this condylar guidance is to program the articulator to accurately mimic the patient's condylar movements saving chairside time spent in correcting occlusal interferences. ${ }^{3}$

The use of a protrusive interocclusal record to program the articulator was suggested by Christensen in 1902. In 1930, Sir Rudolph Hanau introduced the use of hanau formula $\mathrm{L}=\mathrm{H} /$ $8+12$ to measure lateral condylar instructions for adjusting his articulators. These criterias have continued to be used by dentists to program semi adjustable articulators to date. ${ }^{3}$ To that end, the Hanau's formula is still needed for all current hanau models and certain types of articulators. Some models of the hanau articulator may accept lateral interocclusal records, but the hanau fomula has been used for lateral condylar guidance verification even in those models. ${ }^{4}$

Average settings are able to satisfy the demands of treatment and are reliably applicable in a variety of restorations like removable partial dentures, single crowns or short span FPDs. Factors like gender, ethnicity and even age are the roots of extensive controversies on the amount of average values. ${ }^{5-8}$ Sghaireen et al. conducted a study wherein they checked the validity of Hanau's formula in Saudi population and concluded that Hanau formula is not useful as a true predictor of Bennett angle among Saudi population. There is a lot of confusion regarding the condylar inclination values of different races \& ethnicities. There is a lack of uniformity in the literature regarding average condylar inclinations and its comparison to the Hanau formula with respect to an Indian population, therefore this study was undertaken to evaluate and compare 2 methods used to determine the lateral condylar guidance.

\section{METHODS}

The cross sectional in vivo study was carried out in Yenepoya Dental College, Mangalore from June 2020 to December 2020. 24 completely dentulous participants were included in the study. The sample size was calculated using $10 \%$ level of significance and standard deviation 5.99 (from related article) with $2 \%$ of margin of error. Ethical committee approval was obtained before starting the study. All participants to be included in the study had to have all the teeth present.

Participants with any physical or psychological illnesses preventing the attachment of a facebow for registration purposes or the lack of capability to follow or carry out the dentist's instructions correctly or having any dental or periodontal diseases were to be excluded. Impressions of each participant were made using alginate impression material (Tropicalgin ${ }^{\circledR}$ Zhermack $^{\odot}$ ) and casts were poured according to the manufacturer's recommendation.

Protrusive records and lateral records were made for each side using interocclusal recording medium (MAARC ${ }^{\circledR}$ Perfect Bite Registration) (Figure 1, 2 and Figure 3). Facebow transfer was done followed by zeroing of the articulator according to manufacturer's instructions. First the programming of the articulator (HANAU ${ }^{\circledR}$ WIDE VUE, WHIPMIX ${ }^{\circledR}$ ) was done using protrusive record and the horizontal condylar guidance was recorded (Figure 3). The calculated lateral condylar guidance was recorded using the Hanau's formula \& the measured condylar guidance was recorded using right and left lateral records (Figure.4). Both the values obtained were evaluated and compared.

There are various methods for measuring lateral condylar guidance 1) using Hanau's formula ( $\mathrm{L}=\mathrm{h} / 8+12)$, where $\mathrm{L}$ is the lateral condylar guidance and $\mathrm{H}$ is the horizontal condylar guidance and programming the articulator with the values obtained for L. The other method is directly using a lateral interocclusal record wherein the articulator is programmed for L values using the interocclusal records. ${ }^{4}$ So, in this study the groups were classified as Hanau's Formula Left (L), Hanau's Formula Right (R), Interocclusal Records Left (L) \& Interocclusal Records Right (R).

\section{Statistical Analysis}

The Excel and SPSS (SPSS Inc., Chicago, IL, USA) software packages were used for data entry and analysis. The values obtained using the two methods exhibited variation [Graph 1] and were statistically analysed. $\mathrm{T}$ - test for independent samples used at $95 \%$ confidence interval (CI) were used to determine whether statistically significant differences existed between the lateral condylar guidance values obtained using the two methods and values of the right and left sides in both these methods [Table 1]. The $\mathrm{P}<0.05$ was considered statistically significant. 

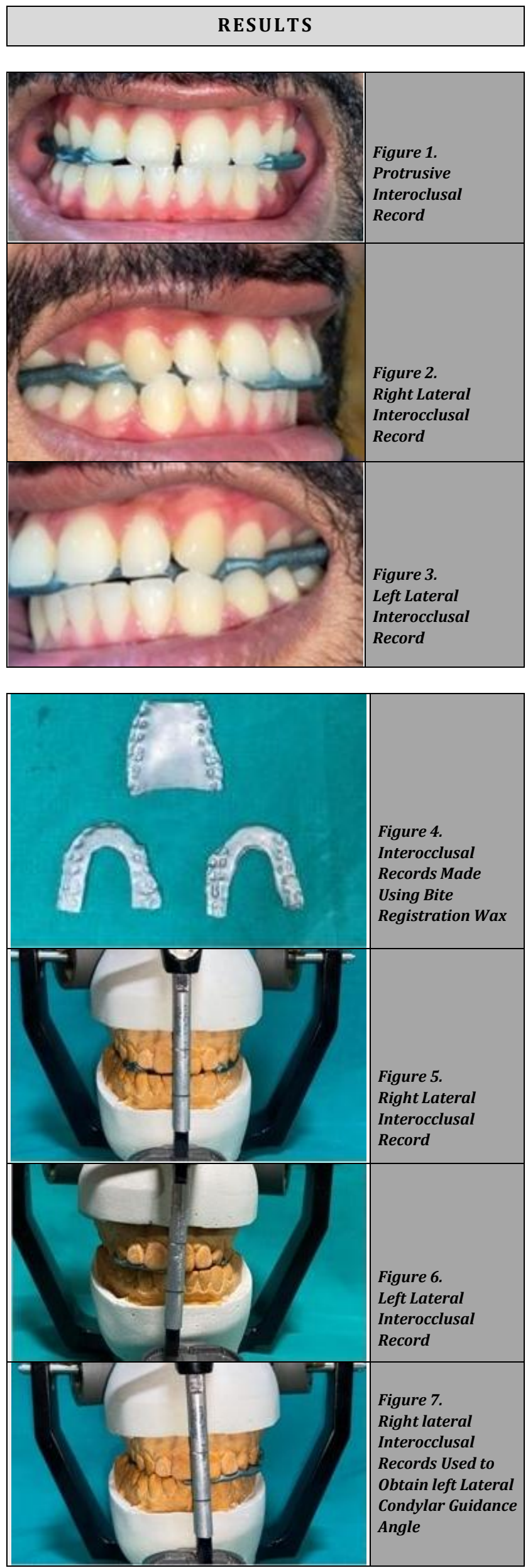

This study included 24 patients ( 10 males and 14 females). Table 1 presents Mean values and Standard Deviation obtained using Hanau's formula and interocclusal records. T-test for independent samples at $95 \% \mathrm{CI}$ was used to determine whether statistically significant differences existed between the lateral condylar guidance values obtained using the two methods. The level of significance was set at $\mathrm{P}=0.05$. The lateral condylar guidance values were obtained using the two methods.The values obtained using Hanau's formula ranged from $14^{\circ}$ to $18^{\circ}$ while those obtained using the interocclusal records ranged from $7^{\circ}$ to $30^{\circ}$. The mean value for lateral condylar guidance obtained using Hanau's Formula on left side was $16.17^{\circ}$ and on the right side was $16^{\circ}$, whereas the mean value for lateral condylar guidance obtained using interocclusal records for the left side was $20.08^{\circ}$ and for the right was $20^{\circ}$. Statistical analysis showed that the lateral condylar guidance values obtained using the Hanau's formula and the interocclusal records had statistically significant differences $(\mathrm{P}<0.05)$ (Table.1).

\begin{tabular}{|ccccc|}
\hline \multicolumn{4}{c}{ Group Statistics } & \\
Group & N & Mean & Std.Deviation & $\begin{array}{c}\text { Std.Error } \\
\text { Mean }\end{array}$ \\
LLCG with Hanau's formula & 24 & 16.17 & 1.404 & .287 \\
LLCG with Interocclusal & 24 & 20.08 & 6.691 & 1.366 \\
$\begin{array}{c}\text { records } \\
\text { RLCG with Hanau's formula }\end{array}$ & 24 & 16.00 & 1.694 & .346 \\
$\begin{array}{c}\text { LLCG with Interocclusal } \\
\text { records }\end{array}$ & 24 & 20.00 & 4.926 & 1.005 \\
\hline
\end{tabular}

Table 1.Comparison of Values of Lateral Condylar Guidance Obtained Using Hanau's Formula and Interocclusal Records

\section{DISCUSSION}

The purpose of this study was to evaluate and compare the lateral condylar guidance values obtained using lateral interocclusal records and Hanau's formula. Only dentulous participants were included as no studies had been done for an Indian population with complete dentition to evaluate the LCG values using different methods.

All conventional diagnostic procedures were followed such as a facebow transfer, zeroing of the articulator and an indirect mounting technique, followed by which the values obtained were compared.

Condylar path is defined as the path traversed by condyle in relation to the articular eminence when the patient's mandible is moved either in protrusive or in the lateral direction from centric relation. Condylar guidance is the mechanical form located in the upper posterior region of an articulator controlling the movement of its mobile member. The Bennett angle and movement can be correlated clinically to the lateral side shift which has an influence on the occlusal morphology of teeth. Setting the condylar guidance of the articulator higher than the subject's relative angle can result in a prosthesis with protrusive and lateral interferences.

The cusps must avoid interfering with the antagonists and must travel along well defined escape routes composed entirely of grooves and cusps. All of this highlights the critical importance of registering and clinically evaluating a precise Bennett angle. The results of this study showed that there was a statistically significant difference between the values obtained using interocclusal records and Hanau's formula. 
Hanau discovered lateral settings regularly varying around $15^{\circ}$, his partner stern maintained that the' formula' was never precise. Hanau claimed that more detailed records could be made once all the teeth were set. He further proposed that to refine the occlusion, remounts and equilibrations were necessary. The engineer did not want the profession to simply set the lateral controls at $15^{\circ}$, indicating a move back to an average value instrument. ${ }^{9}$ The interocclusal records were used as a method to verify the lateral condylar guidance in comparison to lateral condylar guidance obtained using Hanau's formulas as it is relatively inexpensive and a more practically applicable method as compared to a jaw tracking device.

Our study results stand in agreement with those of Javid et al. ${ }^{4}$ Sghaireen et a. ${ }^{10}$ Bhavsar et al. ${ }^{11}$ who reported that Hanau's formula was not an accurate method to obtain lateral condylar guidance. Sghaireen et al. reported the mean values of LCG using Hanau's formula to range between 15 and 18 degrees, ${ }^{10}$ whereas the LCG with interocclusal records ranged between 10 and 17 degrees. Bhavsar et al. reported that the LCG values with Hanau's formula ranged between 14 and 17 degrees whereas the values obtained with jaw tracking device ranged between 8 and 40 degrees. ${ }^{11}$

A larger sample size and a combination of edentulous and dentulous patients could be used to develop an average value for an Indian population for programming the articulator. Further research could be done on dentulous patients and the comparison of LCG values obtained using interocclusal records and LCG values obtained using jaw tracking devices.

Based on the results of this study, dentists and dental technicians should consider reassessing the current recommended average settings and use of the Hanau's formula for programming the semi-adjustable articulators through further research.

\section{CONCLUSIONS}

It may be concluded that the lateral condylar guidance obtained using Hanau's formula is not a precise value and would require time consuming chairside corrections to remove interferences. Thus, it is suggested to use lateral interocclusal records to obtain the lateral condylar guidance for major prosthetic treatments such as long span FPD or full mouth rehabilitation.
Data sharing statement provided by the authors is available with the full text of this article at jemds.com.

Financial or other competing interests: None.

Disclosure forms provided by the authors are available with the full text of this article at jemds.com.

\section{REFERENCES}

[1] The Academy of Prosthodontics Foundation, GPT-9. The glossary of prosthodontic terms. $9^{\text {th }}$ edn. J Prosthet Dent 2017;117(5S):e1-5.

[2] Lundeen HC, Wirth CG. Condylar movement patterns engraved in plastic blocks. J Prosthet Dent 1973;30(6):866-75.

[3] Pelletier LB, Campbell SD. Comparison of condylar control settings using three methods: a bench study. J Prosthet Dent 1991;66(2):193-200.

[4] Javid NS, Porter MR. The importance of the Hanau formula in construction of complete dentures. J Prosthet Dent 1975;34(4):397-404.

[5] Payne JA. Condylar determinants in a patient population: electronic pantograph assessment. J Oral Rehabil 1997;24(2):157-63.

[6] Jasinevicius TR, Pyle MA, Lalumandier JA, et al. The angle of the articular eminence in modern dentate AfricanAmericans and European-Americans. Cranio 2005;23(4):249-56.

[7] Hernandez AI, Jasinevicius TR, Kaleinikova Z, et al. Symmetry of horizontal and sagittal condylar path angles: an in vivo study. Cranio 2010;28(1):60-6.

[8] Alshali RZ, Yar R, Barclay C, et al. Sagittal condylar angle and gender differences. J Prosthodont 2013;22(7):561-5.

[9] Engelmeier RL, Belles DM, Starcke EN. The history of articulators: the contributions of Rudolph L. Hanau and his company-part I. J Prosthodont 2010;19(5):409-18.

[10] Sghaireen MG, Alzarea BK, Kundi I, et al. Application of hanau equation to a Saudi population. International Medical Journal 2020;27(1):95-7.

[11] Bhawsar SV, Marathe AS, Ansari SA. Evaluation of Hanau's formula in determination of lateral condylar guidance: a clinical research study. J Indian Prosthodont Soc 2015;15(4):326-30. 